\title{
Anesthetic variation and potential impact of anesthetics used during endovascular management of acute ischemic stroke
}

\author{
Chitra Sivasankar, ${ }^{1}$ Michael Stiefel, ${ }^{2}$ Todd A Miano, ${ }^{3}$ Guy Kositratna, \\ Sukanya Yandrawatthana, ${ }^{1}$ Robert Hurst, ${ }^{4}$ W Andrew Kofke ${ }^{5}$
}

${ }^{1}$ Department of Anesthesiology and Critical Care, Perelman School of Medicine, University of Pennsylvania, Philadelphia, Pennsylvania, USA

${ }^{2}$ Department of Neurosurgery, WestChester Medical Center, Valhalla, New York, USA ${ }^{3}$ Center for Clinical Epidemiology and Biostatistics, Perelman School of Medicine, University of Pennsylvania, Philadelphia, Pennsylvania, USA

${ }^{4}$ Departments of Radiology, Neurosurgery, and Neurology, Perelman School of Medicine, University of Pennsylvania, Philadelphia, Pennsylvania, USA

${ }^{5}$ Departments of Anesthesiology and Critical Care and Neurosurgery, Perelman School of Medicine, University of Pennsylvania, Philadelphia, Pennsylvania, USA

Correspondence to Professor W Andrew Kofke, Departments of Anesthesiology and Critical Care and Neurosurgery, Perelman School of Medicine, University of Pennsylvania, Philadelphia, PA 19104, USA: kofkea@uphs.upenn.edu

Received 30 July 2015 Revised 29 October 2015 Accepted 4 November 2015 Published Online First 27 November 2015

\section{ABSTRACT}

Background Many authors have reported that general anesthesia (GA), as a generic and uncharacterized therapy, is contraindicated for patients undergoing endovascular management of acute ischemic stroke (EMAIS). The recent American Heart Association update cautiously suggests that it might be reasonable to favor conscious sedation over GA during EMAIS. We are concerned that such recommendations will result in patients undergoing endovascular treatment without consideration of the effects of specific anesthetic agents and anesthetic dose, and without appropriate critical consideration of the individual patient's issues. We hypothesized that significant variation in anesthetic practice comprises $\mathrm{GA}$, and that outcome differences among types of GA would arise.

Methods With IRB approval, we examined the records of patients who underwent anterior circulation EMAIS at the University of Pennsylvania from 2010 to 2015. Patients were managed by different anesthesiologists with no specific protocol. We analyzed American Society of Anesthesiologists status, NIH Stroke Scale, type of stroke, procedure, different types of anesthetic, blood pressure control, and outcome metrics. Modified Rankin Scale (mRS) scores were determined from medical records.

Results GA was used in $91 \%$ of patients. Several types of GA were employed: intravenous, volatile, and intravenous/volatile combined. $\mathrm{mRS}$ scores $\leq 2$ at discharge were observed in $42.8 \%$ of patients receiving volatile anesthesia and were better in patients receiving only volatile agents after induction of anesthesia $(p<0.05)$.

Conclusions Our data support the notion that anesthetic techniques and associated physiology used in EMAIS are not homogeneous, making any statements about the effects of generic GA in stroke ambiguous. Moreover, our data suggest that the type of GA may affect the outcome after EMAIS.

\section{INTRODUCTION}

The primary treatment goal in acute ischemic stroke (AIS) is to reperfuse the brain as quickly and as safely as possible. Endovascular stroke therapy is highly effective in revascularizing patients with AIS. Recently, the MR CLEAN trial (Multicenter Randomized Clinical trial of Endovascular Treatment in the Netherlands) showed significant improvement in primary and secondary outcomes with endovascular therapy compared with medical management. ${ }^{1}$ Van den Berg et al, ${ }^{2}$ investigators in the MR CLEAN trial, published subset analyses on the use of general anesthesia (GA) in the endovascular management of AIS (EMAIS). Their data indicate that patients who had GA showed a worse outcome with endovascular therapy whereas the local anesthesia cohort had improved outcome.

Based on multiple retrospective reports which do not report the specific elements of GA or attempt to adjust results based on the NIH Stroke Scale (NIHSS), authors are now advising not to routinely use GA, although without specific description, for EMAIS. ${ }^{3}{ }^{4}$ Indeed, the recent update published by the American Heart Association ${ }^{4}$ suggests that it might be reasonable to favor conscious sedation over GA during endovascular therapy for AIS, but that prospective studies are warranted. Recommendations generally do acknowledge the non-conclusive retrospective nature of the data. However, we are concerned that non-individualized recommendations for the specific type of anesthesia (uncharacterized GA vs uncharacterized sedation) will result in patients undergoing endovascular thrombectomy without consideration of the specific effects of specific anesthetic agents and without appropriate critical consideration of the individual patient's issues relevant to anesthesia choice.

The purpose of this report is to show the variation in anesthetic practice for EMAIS in our own institution and to compare our results with recent EMAIS trials. Moreover, we test the hypothesis that subsets of anesthetic practice paradigms may impact on neurologic outcome after EMAIS. Lastly, given the ample published speculation regarding mechanisms of GA in worsening of outcome after EMAIS, a secondary aim is to provide a brief overview of potentially important anesthetic effects which may influence the outcome after EMAIS.

\section{METHODS}

We conducted a retrospective cohort study of patients who underwent endovascular treatment of anterior circulation AIS at the University of Pennsylvania from January 2010 to March 2015. We included all patients who underwent endovascular treatment, including mechanical thrombectomy and/or intra-arterial tissue plasminogen activator (tPA). We included patients who had intravenous tPA and endovascular therapy as well as those undergoing endovascular therapy alone, and excluded patients who only received intravenous $\mathrm{tPA}$ or angiography. To assess the 
potential impact of the type of anesthesia exposure on outcome, we divided anesthesia type into four categories: monitored anesthesia care (MAC), total intravenous anesthesia (TIVA), volatile anesthetics, and a combination of intravenous and volatile anesthetics. Clinical outcome was measured using a modified Rankin Scale (mRS) score assigned by two blinded authors after examining the discharge summaries and stroke attending notes as follows: mRS $0-1$, patients who had complete recovery; mRS, mild disability; mRS 3, moderate disability and transferred to rehabilitation; mRS, nursing home with severe disability; mRS 5-6, hospice/withdrawal of care. ${ }^{5}$ Standard monitoring was employed for each patient including pulse oximetry, end-tidal carbon dioxide, oxygen, and anesthetic gas analysis, and ECG. Blood pressure was monitored in each patient by automated sphygmomanometer or by intra-arterial cannula, with efforts generally made to place an arterial cannula but without delaying the intervention.

\section{Data collection}

With IRB approval, we examined the records of patients who underwent anterior circulation EMAIS. Patients were managed by different anesthesiologists with no specific protocol. We analyzed American Society of Anesthesiologists Physical Status (ASA PS), baseline NIHSS, type of stroke, procedure, types of anesthetics, blood pressure control, and outcome metrics.

\section{Statistical analysis}

Baseline characteristics were described with descriptive statistics. Continuous variables are presented as means with SD or medians with IQR as appropriate. Categorical data are presented as numbers with percentages. Univariate analysis of $\mathrm{mRS}$ scores by anesthesia type was performed with the KruskalWallis test followed by Dunn's test using the Bonferroni correction. Blood pressure values were compared between anesthesia types at baseline and various follow-up time points using one-way analysis of variance (ANOVA) followed by pairwise $t$ tests using the Bonferroni correction. A logistic regression model was built for mRS scores $\leq 2$ to control for potential confounding. Variables with significant associations with outcome in univariate logistic regression analysis were included in the multivariable model. All analyses were conducted with Stata V.12, College Station, Texas, USA.

\section{RESULTS}

Eighty-four patients (46 men) of mean age $66 \pm 16$ years were analyzed. Demographic characteristics are shown in table 1.

Table 1 Patient characteristics

\begin{tabular}{lc}
\hline Men & $46(55)$ \\
Age years mean (SD) & $66(16)$ \\
Admission NIHSS & $5(6)$ \\
$\quad<10$ & $39(46)$ \\
$10-19$ & $37(44)$ \\
$20-29$ & $3(4)$ \\
$>30$ & \\
At discharge & $13.0(9)$ \\
Mean (SD) hospital stay, days & $9(11)$ \\
No of patients with tracheostomy & $23(27)$ \\
No of patients with peg & \\
\hline Unless otherwise indicated, data are $n$ (\%). &
\end{tabular}

\section{Specific anesthetic drugs}

Our practice reflects the retrospective and uncontrolled nature of this report. Nonetheless there were distinct differences that allowed us to create groups for comparisons.

Patients in the TIVA group received a combination of propofol bolus and infusion (40-140 $\mu \mathrm{g} / \mathrm{kg} / \mathrm{min})$ and fentanyl boluses; $92 \%$ of patients in this group received a vasopressor.

Patients in the MAC group showed no uniformity with anesthetic practice: four received fentanyl, one received remifentanil only, one received dexmedetomidine, one received propofol; $14 \%$ of patients in this group received a vasopressor.

Patients in the volatile group received $<0.5$ minimum alveolar concentration of desflurane $(>80 \%)$ or sevoflurane $(20 \%)$; $89 \%$ received a vasopressor.

Patients in the combined group received propofol $(30-140 \mu \mathrm{g} /$ $\mathrm{kg} / \mathrm{min}$ ) along with volatile agents at $<0.5$ minimum alveolar concentration; $90 \%$ received a vasopressor.

Of the entire cohort, $26 \%(22 / 84)$ had a mRS score of $0-2$ at discharge; $95 \%$ of these good outcome patients received GA and 5\% received MAC. One patient in the MAC group had a mRS score of $<2$. Several types of GA were employed (table 2). Preinduction/intraprocedure blood pressure monitoring by intra-arterial catheter among the groups was as follows: 35\%/ $94 \%$ for the combined group, 34\%/86\% for the volatile group, $25 \% / 92 \%$ for the TIVA group, and 50\%/50\% for the MAC group. The trend of blood pressure during the procedure is shown in figure 1 , which is notable for parallel trends in systolic, diastolic, and mean arterial blood pressure (MAP) over time. MAP values are further detailed in table 3. MAP values were similar across groups at baseline, and the change at $30 \mathrm{~min}$ and mean MAP during the procedure were also similar. The trend in MAP over time by group is shown in figure 2 . The use of

Table 2 Characteristics of anesthesia groups

\begin{tabular}{lllll}
\hline & MAC & TIVA & Vol & Comb \\
\hline No of patients & 7 & 12 & 35 & 30 \\
Men & $1(14.3)$ & $5(41.6)$ & $22(62.8)$ & $18(60)$ \\
Age, mean (SD) & $75.0(11.4)$ & $69.8(15.9)$ & $62.7(17.2)$ & $66.5(15.3)$ \\
Hypertension & $7(100)$ & $11(91.6)$ & $27(77.1)$ & $27(90)$ \\
Diabetes mellitus & $2(28.5)$ & $5(41.6)$ & $9(25.7)$ & $7(23.3)$ \\
Atrial fibrillation & $5(71.4)$ & $7(58.3)$ & $20(57.1)$ & $17(56.6)$ \\
Hyperlipidemia & $7(100)$ & $9(75)$ & $21(60)$ & $22(73.3)$ \\
Prior stroke/TIA & $1(14.2)$ & $2(16.6)$ & $4(11.4)$ & $7(23.3)$ \\
Coronary artery disease & $6(85.7)$ & $9(75)$ & $21(60)$ & $21(70)$ \\
NIHSS, median (IQR) & $18(16-22)$ & $21.5(16-26)$ & $19(13-22)$ & $19(14-23)$ \\
Proximal occlusion M1 & $4(57.1)$ & $6(50)$ & $19(54.2)$ & $14(46.6)$ \\
M2 occlusion & $2(28.5)$ & $0(0.0)$ & $8(22.8)$ & $4(13.3)$ \\
ICA occlusion & $1(14.2)$ & $6(50)$ & $8(22.8)$ & $12(40)$ \\
Mechanical thrombectomy & $5(71.4)$ & $10(83.3)$ & $30(85.7)$ & $26(86.6)$ \\
IA tPA & 0 & $7(58.3)$ & $13(37.1)$ & $10(33.3)$ \\
Combination* & 0 & $5(41.6)$ & $10(28.5)$ & $9(30)$ \\
ASA PS score <3 & $3(42.9)$ & $4(33.3)$ & $13(37.1)$ & $14(46.7)$ \\
Any vasopressor support & $1(14.3)$ & $11(91.7)$ & $31(88.6)$ & $27(90.0)$ \\
Good recovery (mRS 0-2) & $1(14.3)$ & $3(25)$ & $15(42.9)$ & $3(10)$ \\
\hline Unless noted ofherwise, & & & &
\end{tabular}

Unless noted otherwise, data are $\mathrm{N}(\%)$.

*Patients undergoing both mechanical thrombectomy and intra-arterial tPA. ASA PS, American Society of Anesthesiologists Physical Status; Comb, combined intravenous and volatile anesthesia; IA, intra-arterial; MAC, monitored anesthesia care; mRS, modified Rankin Scale; NIHSS, NIH stroke scale; TIA, transient ischemic attack; TIVA, total intravenous anesthesia; TPA, tissue plasminogen activator; Vol, volatile. 


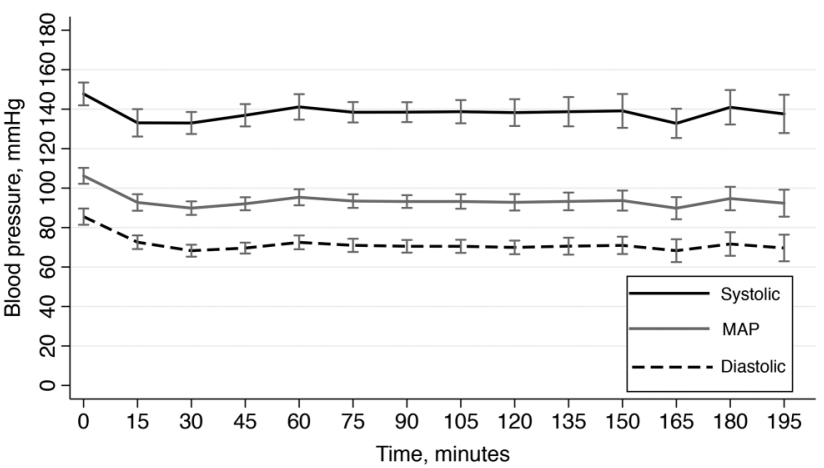

Figure 1 Trend of blood pressure over time in all patients. Error bars represent the $95 \% \mathrm{Cl}$ for each measure at each time point.

vasopressor drugs to support blood pressure was common in the population, with the exception of the MAC group (table 2). Of the patients who received any vasopressor support, 55/70 (78.6\%) received continuous infusions of either phenylephrine $(n=50)$ or epinephrine $(n=5)$. The remaining 15 patients received only bolus therapy with either phenylephrine $(n=7)$ or ephedrine $(n=8)$.

In univariate analysis, mRS scores differed significantly across anesthesia type, with the best outcome and lowest mRS scores observed in patients who received only volatile agents after induction of anesthesia $(\mathrm{p}<0.05)$ (figure 3$)$. Other significant predictors of the mRS score in univariate analysis were NIHSS and age. mRS was not associated with any blood pressure parameter (baseline, change at $30 \mathrm{~min}$, or mean during procedure; $p>0.05$ for all comparisons). The trend in MAP stratified by outcome status is shown in figure 4. In addition, mRS was not associated with the use of vasopressor drugs to support blood pressure. Importantly, the high rate of vasopressor use in the population limits the power to examine this relationship. Variables with significant univariate associations, along with anesthesia type, were included in a multivariable logistic regression model. After adjusting for NIHSS and age, anesthesia type remained significantly associated with the mRS score at discharge (table 4). Further adjustment for any of the MAP parameters did not alter the results of the final regression model.

\section{DISCUSSION}

Two major conclusions of our evaluation of our institutional anesthesia practice for EMAIS are apparent: (1) in the absence of a specific protocol, significant variation in anesthetic management can arise; and (2) the type of general anesthetic used may affect the outcome.

In a non-protocol environment with anesthesia at night often provided by in-house on-call non-neuro-oriented anesthesiology teams, significant variation in anesthesia was observed but with reasonably tight control of blood pressure. John et $a l^{6}$ also noted variation in a report of their use of conscious sedation. This suggests that reference to non-characterized generic GA or sedation as an element of EMAIS reports may be inappropriate and lead authors to invalid or ambiguous conclusions. Anesthesiologists evaluating such reports and faced with a patient in need of EMAIS are uncertain as to what is being recommended to be used or avoided. Future reports about management of EMAIS patients should address specific anesthetic and physiologic details. Notably, a proposed prospective investigation, the SIESTA trial, continues this pattern of studying
Table 3 Mean arterial blood pressure

\begin{tabular}{|c|c|c|c|c|}
\hline & MAC & TIVA & Vol & Comb \\
\hline \multicolumn{5}{|l|}{ MAP } \\
\hline Baseline $^{a}$ & $103.3(28.1)$ & $102.0(25.5)$ & $109.5(17.7)$ & 104.8 (13.7) \\
\hline $\begin{array}{l}\text { Maximum* } \\
\text { change over } \\
\text { first } 30 \mathrm{~min}^{\mathrm{b}}\end{array}$ & $-16.6(7.6)$ & $-19.0(22.2)$ & $-24.8(17.8)$ & $-19.2(14.4)$ \\
\hline $\begin{array}{l}\text { Mean MAP } \\
\text { during } \\
\text { procedure }^{c}\end{array}$ & $95.1(16.2)$ & $89.0(10.8)$ & $95.8(12.2)$ & $94.3(11.0)$ \\
\hline \multicolumn{5}{|c|}{$\begin{array}{l}{ }^{a} p=0.31 ;{ }^{b} p=0.53 ;{ }^{c} p=0.41 \text {. } \\
{ }^{*} \text { This value represents the maximum observed change in MAP during the initial } \\
30 \text { min of the procedure. } \\
\text { Comb, combined intravenous and volatile anesthesia; MAC, monitored anesthesia } \\
\text { care; MAP, mean arterial blood pressure; TIVA, total intravenous anesthesia; Vol, } \\
\text { volatile. }\end{array}$} \\
\hline
\end{tabular}

uncharacterized GA without involvement of anesthesia teams or coinvestigators. ${ }^{7}$

Our data indicate that the type of anesthesia employed may indeed be predictive or associated with outcome in EMAIS, as the various available anesthetic techniques and drugs have disparate neurochemical, neurophysiologic, and systemic effects. ${ }^{8} 9$ Because of this concern, we divided our retrospective dataset into four general categories of anesthesia: MAC, volatile, TIVA, and combined TIVA and volatile. Even this division is potentially confusing as there are multiple volatile agents, which have some differences between them, and numerous intravenous agents are used in TIVA, which have disparate mechanisms and neurophysiologic effects. Moreover, there are even more differences related to the non-linear effects of depth of anesthesia on metabolism ${ }^{810}$ or dose-related potential neuroprotection/neurotoxicity. ${ }^{11}$ Our data suggest that attention to the elements of GA matter. Indeed, we made the unexpected observation of improved outcome with the use of volatile anesthetics.

As with every retrospective study, this observation is the hypothesis for a future prospective study. The sample size is rather small, the distribution of internal carotid artery occlusions was not homogeneous, we did not record Thrombolysis In Cerebral Infarction scores which arguably may be affected by concurrent anesthetic, and assigning mRS scores based on the medical record has been criticized, ${ }^{12}$ making comparison with other studies suspect but nonetheless probably useful within a single study. There is a potentially significant possibility that this finding of a favored technique is born simply of chance or related to different demographics, stroke severity, or medical conditions. It would thus be inappropriate to advise any specific anesthesia practice based on our observations.

Our investigation raises important questions regarding what aspects of periprocedural management actually contribute to the end result. Given the ample speculations in EMAIS reports on the potential mechanisms of the impact of GA on outcome after EMAIS, we will briefly review what is known and relevant to the ischemic brain about effects of the disparate anesthetic techniques that we employed in EMAIS.

\section{ASA PS versus NIHSS}

As NIHSS assesses stroke severity only and does not assess patients' overall medical well-being related to anesthesia outcome, its use could produce misleading conclusions when analyses use NIHSS to correct for severity of baseline illness in analyzing the effects of anesthesia on a neurologic process. This implies that the use of NIHSS as a measure to correct for 
Figure 2 Trend of mean arterial blood pressure over time stratified by anesthesia type. Error bars represent the $95 \% \mathrm{Cl}$ for each measure at each time point. No significant differences were noted between groups across all time points.

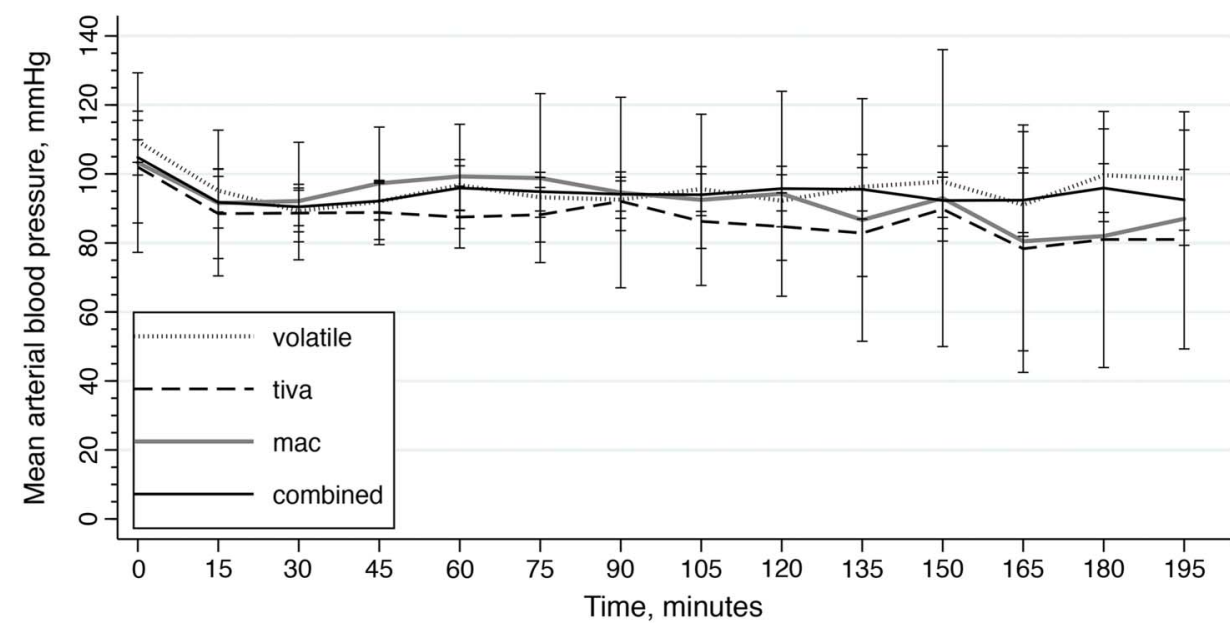

baseline status in evaluating the effects of anesthesia, as is done in many reports, is a suboptimal use of a scale for which it was not designed. ASA PS assesses the overall health and function of the patient including cardiac and respiratory status, and it incorporates non-neurologic issues not included in the NIHSS. However, ASA PS is imperfect as it is low on detail. Perhaps common ICU scoring systems such as APACHE (Acute Physiology and Chronic Health Evaluation), MPM (Mortality Probability Model), or SAPS (Simplified Acute Physiology Score)-three major ICU scoring and risk adjustment systems in use $^{13} 14$-would be more useful when stroke studies are correcting for the impact of systemic factors on the role of anesthesia in neurologic outcome. Anesthesia affects every organ in the body but NIHSS assesses only baseline neurologic function.

\section{Blood pressure management}

Blood pressure management is thought to be an essential element of EMAIS, but most reports do not include blood pressure data. Given that GA clearly predisposes to lower blood pressure, possibly contributing to a bad outcome, ${ }^{15-17}$ this is certainly a valid area of concern. Conversely, John et $a l^{6}$ noted a worse outcome with GA in EMAIS but detected no difference in blood pressure between sedation and GA. Takahashi et al ${ }^{18}$ noted decreased blood pressure with GA but no effect on outcome after EMAIS, and Jagani et $a l^{16}$ reported significant blood pressure effects of GA but did not observe a link between blood pressure and outcome. On the other hand, Mundiyanapurath et $a l^{19}$ maintained blood pressure but found an inverse association between the amount of norepinephrine

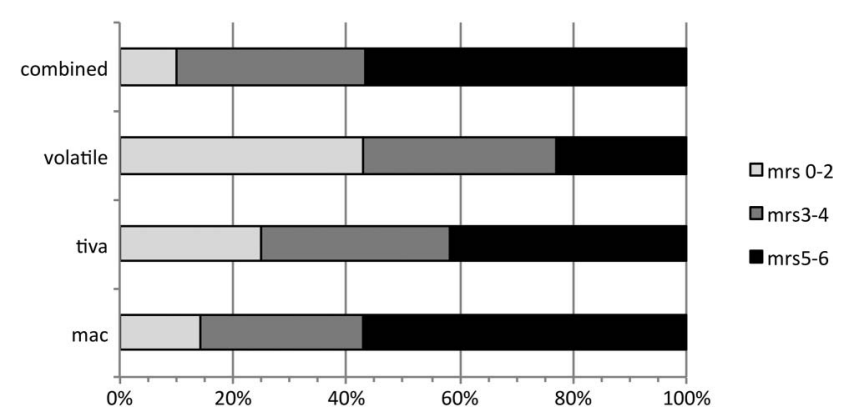

Figure 3 Outcome metrics. Percentage of patients with modified Rankin Scale (mRS) scores at discharge derived from the medical record, according to type of anesthesia. Scores were better in the group receiving volatile anesthesia after induction of anesthesia $(p<0.05)$. used and outcome. These observations provide a hint that the real issue is the dose of anesthetic or sedative, as higher GABAergic anesthetic dose in general may produce a greater decrement in blood pressure and/or a higher pressor requirement. Conversely, anesthetic agents which do not decrease blood pressure so much-for example, opioids, ketamine, nitrous oxide-have neurotoxic potential, as discussed below. Unfortunately, these reports on the impact of GA on EMAIS lack details on the anesthetic drugs employed.

Notably, in our series, a blood pressure effect between anesthetic or between outcome groups was not apparent. Notwithstanding the lack of a protocol, this suggests a general appreciation among our anesthesiologists of the importance of blood pressure management during ischemic stroke. Indeed, we may also be revealing the importance of having anesthesiologists involved in the management of these patients. It is noteworthy that such involvement of anesthesiologists is not included in the methods sections in many reports. ${ }^{2} 71719$ Importantly, the report by Jagani et $a^{16}$ showing a worse outcome with GA and lower blood pressure indicates a large percentage of patients receiving volatile anesthesia, with nitrous oxide (potentially neurotoxic $^{11}$ ) in $11 \%$ and propofol infusion in $11 \%$. No subgroup analysis similar to ours or description of anesthetic doses is provided. Patients in our volatile anesthesia group received doses expected to be amnestic but well below the minimum alveolar concentration, a dose which would typically be used for more invasive procedures. The end-tidal anesthetic concentration monitoring allows for precise titration of dose to end-organ concentration. Conversely, intravenous propofol infusions do not provide that capability and, if not appropriately titrated to clinical signs of anesthetic depth, tend to develop progressively higher brain and blood concentrations over time, ${ }^{20}$ with expected adverse hemodynamic consequences. The report by Löwhagen Hendén et $a l^{17}$ clearly indicates an association between GA and low blood pressure and worse outcome but, similar to many other reports, gives no details on the composition of GA nor whether there was anesthesiologist involvement. Overall, it is apparent that conclusive information is either absent or conflicting regarding the potential role of blood pressure and its relationship to anesthetic dose in the observed effects of GA on outcome after EMAIS.

A variety of anesthetic drugs were employed in our patients. Thus, some comment on the disparate neurologic effects of these drugs is relevant.

\section{Anesthetic drugs}


Figure 4 Trend of mean arterial blood pressure over time stratified by outcome defined by modified Rankin Scale (mRS) score: good outcome defined as mRS score $\leq 2$; bad outcome defined as $\mathrm{mRS}$ score $>2$. Error bars represent the $95 \% \mathrm{Cl}$ for each measure at each time point. No significant differences were noted between groups across all time points.

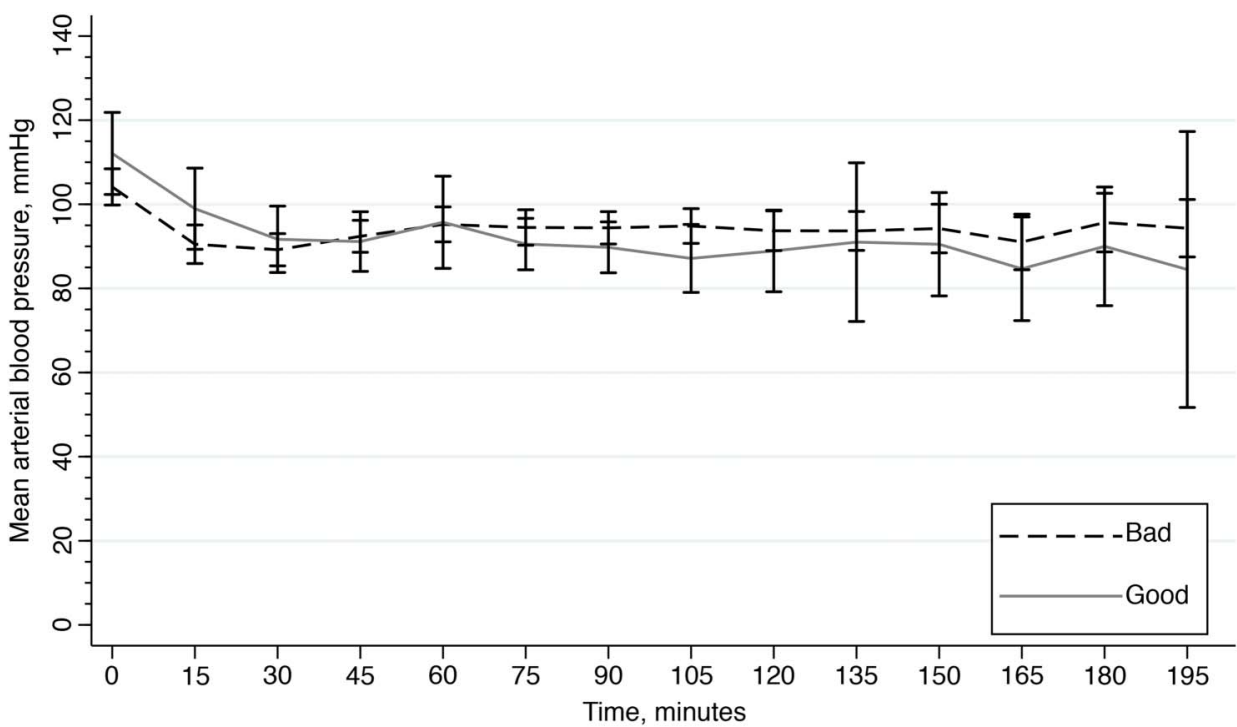

Volatile anesthetic agents

Inhaled anesthetic agents (eg, isoflurane) have protean neurologic effects. They enhance inhibitory synaptic transmission by enhancing GABA and glycine and concurrently inhibit excitatory NMDA-type glutamate and neuronal nicotinic acetylcholine receptors, activation of two-pore domain $\mathrm{K}_{2 \mathrm{P}}$ channels and leak $\mathrm{K}^{+}$channels. ${ }^{9}$

In general, inhalational agents uncouple metabolism from cerebral blood flow $(\mathrm{CBF})$ in a dose-related manner, increasing $\mathrm{CBF}$ while reducing metabolic rate (CMR) in a non-linear manner, which becomes more apparent with increasing dose. ${ }^{9}$ Although deeper anesthesia might be predicted to be neuroprotective, preclinical studies suggest this may not be the case. ${ }^{21}$ There is much discussion in EMAIS reports that volatile anesthetics induce cerebral vasodilation and risk an intracranial steal. The possibility of a steal has been suggested with carotid occlusion during carotid endarterectomy. ${ }^{22}$ However, higher brain tissue oxygen tension was reported with volatile agents compared with intravenous anesthetics, ${ }^{23}$ supporting the notion that volatile anesthetic-mediated vasodilation may be beneficial. Moreover, volatile agents used in the context of carotid endarterectomy decrease the threshold low CBF at which electroencephalography changes arise, but with somewhat unexplainable differences between agents, nonetheless further supporting a potential contribution to neuroprotection during EMAIS. ${ }^{24}$

Table 4 Multivariable logistic regression model for achieving mRS score $\leq 2$ at discharge

\begin{tabular}{llll}
\hline Variable & OR & $95 \% \mathrm{Cl}$ & $\mathbf{p}$ Value \\
\hline $\begin{array}{l}\text { Anesthesia type* } \\
\text { Volatile }\end{array}$ & reference & & 0.048 \\
TIVA & 0.69 & 0.13 to 3.77 & \\
MAC & 0.35 & 0.04 to 3.76 & \\
Comb & 0.15 & 0.04 to 0.66 & \\
Age & 0.96 & 0.93 to 0.99 & 0.044 \\
NIHSS & 0.87 & 0.79 to 0.96 & 0.010 \\
\hline
\end{tabular}

* Reference category for anesthesia type is volatile. Thus, ORs for the other anesthesia types are in reference to volatile anesthesia.

Comb, combined intravenous and volatile anesthesia; MAC, monitored anesthesia care; mRS, modified Rankin Scale; NIHSS, NIH Stroke Scale; TIVA, total intravenous anesthesia.
Intravenous anesthetic agents

Commonly used modern intravenous anesthetic agents in the USA include the GABAergic drugs propofol, barbiturates, etomidate, and benzodiazepines. Other intravenous drugs with different neurochemical mechanisms include ketamine, opioids, and dexmedetomidine. Thiopental is available only outside the USA.

\section{GABAergic drugs: propofol, thiopental, etomidate, and benzodiazepines}

These drugs bind to the GABA receptor and increase conductivity to chloride ions leading to hyperpolarization of cell membranes. ${ }^{9}$ They decrease CBF coupled to decreased $\mathrm{CMR}^{9}$ and can be expected to increase $\mathrm{CBF}$ to an ischemic area. Barbiturate neuroprotection was reported in the context of focal temporary ischemia in a subhuman primate model. ${ }^{25}$ The similarity of this to EMAIS suggests that barbiturates or propofol should be helpful in EMAIS. However, the lower brain oxygen tension relative to volatile agents ${ }^{23}$ and our results suggest otherwise.

\section{Opioids}

Commonly used anesthetic opioids act predominantly on $\mu$-receptors. ${ }^{8}{ }^{9}$ Effects on CMR and CBF are variable, dosedependent, and affected by other background anesthetics. Hemodynamically, they can cause bradycardia but with minimal potential for hypotension. No neuroprotection has been reported with opioids, although at high but clinically relevant doses in rodents they produce limbic seizure, hypermetabolism, and brain damage with congruent neurometabolic effects in humans.

\section{CONCLUSIONS}

Overall, this summary of anesthetic effects and our data indicate that anesthetics can increase or decrease CBF and CMR and confer both neuroprotection and neurotoxicity. Given that reports that GA worsens the outcome in EMAIS do not describe the specific anesthetics involved or doses, and that our data suggest anesthetic techniques and drugs employed are heterogeneous and may affect the outcome in a drug-specific manner, we believe that current data provide inadequate guidance for stroke teams (including the anesthesiologists) regarding the specific choice of sedation or general anesthetic drugs to use during 
EMAIS. Our retrospective data suggest that, when GA is selected, maintenance with volatile anesthetics alone may be associated with improved outcome, but this requires prospective confirmation.

Contributors CS, WAK: made substantial contributions to the conception or design of the work or the acquisition and interpretation of data for the work, drafted the work and revised it critically for important intellectual content, provided final approval of the version to be published, and agree to be accountable for all aspects of the work in ensuring that questions related to the accuracy or integrity of any part of the work are appropriately investigated and resolved. MS: made substantial contributions to the conception or design of the work and interpretation of data for the work, provided critical insights in the drafting of the manuscript for important intellectual content, provided final approval of the version to be published, and agrees to be accountable for all aspects of the work in ensuring that questions related to the accuracy or integrity of any part of the work are appropriately investigated and resolved. TAM: made substantial contributions to the analysis and interpretation of data for the work, provided critical insights in the drafting of the manuscript for important intellectual content, provided final approval of the version to be published, and agrees to be accountable for all aspects of the work in ensuring that questions related to the accuracy or integrity of any part of the work are appropriately investigated and resolved. GK, SY: made substantial contributions to the acquisition and interpretation of data for the work, participated in drafting the work and revising it critically for important intellectual content, provided final approval of the version to be published, and agree to be accountable for all aspects of the work in ensuring that questions related to the accuracy or integrity of any part of the work are appropriately investigated and resolved. RH: made substantial contributions to the conception of the work, interpretation of data for the work, participated in drafting the work and revising it critically for important intellectual content, provided final approval of the version to be published, and agrees to be accountable for all aspects of the work in ensuring that questions related to the accuracy or integrity of any part of the work are appropriately investigated and resolved.

Funding This work was supported by National Heart, Lung, and Blood Institute of the National Institutes of Health, grant number 1F32HL124914-01 through support of Dr Miano.

Competing interests None declared.

Ethics approval Ethics approval was obtained from University of Pennsylvania Biomedical IRB 7, protocol number 822224.

Provenance and peer review Not commissioned; externally peer reviewed.

Data sharing statement Deidentified summary data are available upon request of the corresponding author.

\section{REFERENCES}

1 Berkhemer OA, Fransen PSS, Beumer D, et al. A randomized trial of intraarterial treatment for acute ischemic stroke. N Eng/ J Med 2015;372:11-20.

2 van den Berg LA, Koelman DLH, Berkhemer OA, et al. Type of anesthesia and differences in clinical outcome after intra-arterial treatment for ischemic stroke. Stroke 2015;46:1257-62.

3 Grotta JC, Hacke W. Stroke Neurologist's perspective on the new endovascular trials. Stroke 2015;46:1447-52.

4 Powers WJ, Derdeyn CP, Biller J, et al. 2015 AHA/ASA focused update of the 2013 guidelines for the early management of patients with acute ischemic stroke regarding endovascular treatment: a guideline for healthcare professionals from the American Heart Association/American Stroke Association. Stroke 2015;46:3020-35.

5 Kasner SE. Clinical interpretation and use of stroke scales. Lancet Neurol 2006:5:603-12

6 John S, Thebo U, Gomes J, et al. Intra-arterial therapy for acute ischemic stroke under general anesthesia versus monitored anesthesia care. Cerebrovasc Dis 2014;38:262-7

7 Schönenberger S, Möhlenbruch M, Pfaff J, et al. Sedation vs. Intubation for Endovascular Stroke TreAtment (SIESTA) —a randomized monocentric trial. Int J Stroke 2015:10:969-78.

8 Brown EN, Purdon PL, Van Dort CJ. General anesthesia and altered states of arousal: a systems neuroscience analysis. Annu Rev Neurosci 2011; 34:601-28.

9 Sloan TB. Anesthetics and the brain. Anesthesiol Clin North Am 2002;20:265-92.

10 Stullken EH Jr, Milde JH, Michenfelder JD, et al. The nonlinear responses of cerebral metabolism to low concentrations of halothane, enflurane, isoflurane, and thiopental. Anesthesiology 1977;46:28-34.

11 Jevtović-Todorović V, Todorović S, Mennerick S, et al. Nitrous oxide (laughing gas) is an NMDA antagonist, neuroprotectant and neurotoxin. Nature Med 1998:4:460-3.

12 Quinn TJ, Ray G, Atula S, et al. Deriving modified Rankin scores from medical case-records. Stroke 2008;39:3421-3.

13 Breslow MJ, Badawi 0. Severity scoring in the critically ill. Part 2: maximizing value from outcome prediction scoring systems. Chest 2012;141:518-27.

14 Breslow MJ, Badawi 0. Severity scoring in the critically ill. Part 1: interpretation and accuracy of outcome prediction scoring systems. Chest 2012;141:245-52.

15 Davis MJ, Menon BK, Baghirzada LB, et al. Anesthetic management and outcome in patients during endovascular therapy for acute stroke. Anesthesiology 2012:116:396-405.

16 Jagani M, Brinjikji W, Rabinstein AA, et al. Hemodynamics during anesthesia for intra-arterial therapy of acute ischemic stroke I Neurointerv Surg. Published Online First: 14 Sep 2015.

17 Löwhagen Hendén P, Rentzos A, Karlsson J-E, et al. Hypotension during endovascular treatment of ischemic stroke is a risk factor for poor neurological outcome. Stroke 2015;46:2678-80

18 Takahashi CE, Brambrink AM, Aziz MF, et al. Association of intraprocedural blood pressure and end tidal carbon dioxide with outcome after acute stroke intervention. Neurocrit Care 2014;20:202-8.

19 Mundiyanapurath S, Stehr A, Wolf M, et al. Pulmonary and circulatory parameter guided anesthesia in patients with ischemic stroke undergoing endovascular recanalization J Neurointerv Surg 2016;8:335-41.

20 Schüttler J, Ihmsen $\mathrm{H}$. Population pharmacokinetics of propofol: a multicenter study. Anesthesiology 2000;92:727-38.

21 Warner D, Takaoka S, Wu B, et al. Electroencephalographic burst suppression is not required to elicit maximal neuroprotection from pentobarbital in a rat model of focal cerebral ischemia. Anesthesiology 1996;84:1475-84.

22 McCulloch TJ, Thompson CL, Turner MJ. A randomized crossover comparison of the effects of propofol and sevoflurane on cerebral hemodynamics during carotid endarterectomy. Anesthesiology 2007;106:56-64.

23 Hoffman WE, Charbel FT, Edelman G, et al. Thiopental and desflurane treatment for brain protection. Neurosurgery 1998:43:1050-3.

24 Michenfelder J, Sundt T, Fode N, et al. Isoflurane when compared to enflurane and halothane decreases the frequency of cerebral ischemia during carotid endarterectomy. Anesthesiology 1987;67:336.

25 Selman W, Spetzler R, Roessmann U, et al. Barbiturate-induced coma therapy for focal cerebral ischemia. Effect after temporary and permanent MCA occlusion. J Neurosurg 1981;55:220. 